\title{
Quality of Life in Actual Living Liver Donors Versus Potential Living Liver Donors
}

\author{
Zeeshan Butt, PhD $^{1,2,3,{ }^{*} \text {, Daniela Ladner, MD, MPH }}{ }^{1,2,3}$, and Neehar Parikh, MD ${ }^{1,4}$ \\ ${ }_{1}^{1}$ Northwestern University Transplant Outcomes Research Collaborative (NUTORC), \\ Comprehensive Transplant Center, Northwestern University, Chicago IL \\ 2 Department of Medical Social Sciences, Northwestern Feinberg School of Medicine, Chicago IL \\ 3 Institute for Healthcare Studies, Northwestern Feinberg School of Medicine, Chicago IL \\ ${ }^{4}$ Division of Gastroenterology, Department of Medicine, Northwestern Feinberg School of \\ Medicine, Chicago IL
}

To the Editors:

We read with great interest the article by Schulz and colleagues1, who reported on early findings from their prospective, multicenter study of quality of life in actual living liver donors and a control group of potential living liver donors. To our knowledge, theirs is the first published prospective study of living liver donor quality of life that has used a control group. However, there are some methodological concerns that would seem to prevent drawing strong conclusions from their data.

Our first concern relates to the composition of the control group. The authors reported that 149 of 233 (64\%) individuals were evaluated at baseline, with 61 being actual donors and 88 being potential donors. At the 3 month follow-up, data were available from $43(70 \%)$ actual donors and $46(52 \%)$ potential donors. However, additional exclusionary criteria resulted in dropping data from 13 potential donors, resulting in an effective retention rate of $38 \%$. While there are many attractive aspects of using potential donors as controls for living donors, even in this relatively small sample, the differential rate of attrition between the study groups gives reason for pause. Analysis of the patient-reported outcome data was conducted on the subset of actual and potential donors with data at baseline and the 3-month follow-up. While the authors reported that the analyzed groups of actual versus potential donors were relatively similar with respect to sociodemographics, it is not clear whether the potential donors that were included differ from the ones that dropped out of the study with respect to sociodemographics or quality of life. While the authors note this problem in their discussion, this remains an open question that can and should be addressed empirically. Having the answer to this question would be necessary prior to generalizing the Schulz et al findings in any meaningful way.

Our second concern relates to the current practice of using patient-reported outcomes in living liver donation studies, more generally. Certainly, such scales will be necessary for ongoing research in this important area of investigation. However, we are aware of no scales that have been developed to address the concerns identified as being most germane by living liver donors themselves. Until such scales are developed, researchers often resort to using

*Please address correspondence to: Zeeshan Butt, PhD, Departments of Medical Social Sciences and Surgery, Northwestern University Feinberg School of Medicine, 750 N. Lake Shore Drive, $10^{\text {th }}$ Floor, Chicago, IL 60611 (USA), 312-503-5519, phone, z-

butt@northwestern.edu. 
generic scales (such as the SF-36) or disease-specific scales, which are typically intended to assess patients with end stage organ disease (e.g. Schulz and colleagues' use of the Chronic Liver Disease Questionnaire). This practice may or may not be appropriate for otherwise healthy donor populations, and it is necessary to establish the reliability and validity of disease-specific scales when used in related, but off-target populations. Similarly, while it is somewhat informative to report on statistically significant differences between groups or over time in the same group, there is a need to place such differences in context by addressing the clinical significance of such changes. This latter criticism is not specific to the Schulz et al report, but is a more general call for increased attention to the use and interpretation of patient-reported outcomes in future organ donation and transplantation research.

\section{Acknowledgments}

Supported in part by grant UL1RR025741 from the National Center for Research Resources, National Institutes of Health.

\section{References}

1. Schulz KH, Kroencke S, Beckmann M, Nadalin S, Paul A, Fischer L, et al. Mental and physical quality of life in actual living liver donors versus potential living liver donors: A prospective, controlled, multi-center study. Liver Transpl. 2009; 15:1676-1687. [PubMed: 19938145] 